\title{
Prevalence and antimicrobial susceptibility of anaerobes from patients with periodontal abscess in China
}

\author{
Junlin $\mathrm{He}^{1,4}$, Qing Chang ${ }^{1,4}$, Fupin $\mathrm{Hu}^{2}$, Xiping Feng ${ }^{3}$, Demei $\mathrm{Zhu}^{2}$ and Liying $\mathrm{Yu}^{1}$ \\ The Journal of Antibiotics (2013) 66, 97-98; doi:10.1038/ja.2012.94; published online 21 November 2012
}

Keywords: anaerobe; antimicrobial susceptibility testing; periodontal abscess

Periodontal abscess is defined as a localized purulent infection affecting the tissues surrounding a periodontal pocket that can lead to the destruction of supporting structures. ${ }^{1}$ The treatment for periodontal abscesses includes drainage through the pocket or an incision. The systemic administration of antibiotics can serve as an adjunct to mechanical therapy. ${ }^{2}$ In the clinic, the administration of antibiotics depends mainly on the doctor's personal experience, and may result in the emergence of a large number of drug-resistant strains. ${ }^{3,4}$ Temporal and geographic changes in antibiotic susceptibility among anaerobes have been reported, ${ }^{5}$ and antibiotic-resistant anaerobic bacteria have become increasingly recognized as a confounding factor in the selection of therapeutic agents. ${ }^{6-8}$ In this respect, it is necessary to provide a reasonable evidence for the clinical use of antimicrobial agents by analyzing the distribution and drug resistances of anaerobes collected from patients with periodontal abscesses. A prospective study including 135 patients (72 males and 63 females, aged 30-64) with no current usage of medication (about 3 months) was carried out from June 2007 to January 2009. Patients with systemic disease or periodontal therapy were excluded. The anaerobes were isolated on Wilkins-Chalgren Agar (Oxoid, Hampshire, UK) supplemented with $5 \%$ sheep blood and vitamin $\mathrm{K}_{1}$ in an anaerobic box (Biomerieux, Lyon, France). After incubation for $48 \mathrm{~h}$, colony morphology, fluorescence and pigment characteristics were studied, and anaerobic bacteria were identified at the species level by the API-32A system (Biomerieux) according to the manufacturer's instructions. Antimicrobial susceptibilities of the bacteria were determined by the agar dilution method. Bacteroides fragilis ATCC 25285 served as a control strain. SPSS 19.0 software (Chicago, IL, USA) was used for statistical analysis. The results are shown in Table 1.

A periodontal abscess is an acute lesion, resulting in the rapid destruction of tooth support structures, and periodontal bacterial infection is one of the major pathogenic factors. ${ }^{9}$ Previous studies have demonstrated that seven pathogenic anaerobes, namely Porphyromonas gingivalis, B. forsythus, Prevotella intermedia, Peptostreptococcus micros, Fusobacterium nucleatum, Actinobacillus actinomycetemcomitans and Prevotella melaninogenica, exist in the subgingival plaque of patients with periodontal disease. ${ }^{10,11}$ As reported, the microflora of periodontal abscesses is characterized by the presence of periodontal pathogens, and black-pigmented bacteria (P. gingivalis and P. melaninogenica) were found as the most prevalent group of bacteria. ${ }^{12}$ In our study, among 81 strains $P$. gingivalis and P. melaninogenica accounted for $3.7 \%$ and $83.9 \%$ respectively, demonstrating similar presence of these pathogenic bacteria in periodontal abscesses patients. The results of antimicrobial susceptibility testing indicated that all isolates were sensitive to imipenem, and $88.2 \%$ of 81 isolates were sensitive to cefoxitin, similar to observations in other studies. ${ }^{13,14}$ The rates of resistance to chloramphenicol and metronidazole were $19.2 \%$ and $24.5 \%$, respectively. Only $22.8 \%$ of the isolates were sensitive to clindamycin, an observation that is comparable to that of other studies, ${ }^{15}$ indicating a relatively high resistance rate. The results of the antimicrobial susceptibility testing demonstrated that the resistance rate to metronidazole, which is commonly used for the treatment of infection due to anaerobes, was increasing. Metronidazole is mainly active against anaerobes, and is prescribed frequently in Chinese dental clinics. However, with the misuse of antibiotics and the increased variability of bacteria, bacterial resistance to metronidazole is becoming more frequent. In our study, the resistance rate of 81 isolates to metronidazole (24.5\%) was higher than that in the report by Maestre et al. ${ }^{16}(<6 \%)$. Therefore, it is important to investigate the susceptibility of anaerobes to antibiotics, and perform further studies to clarify the mechanism of antibiotic

\footnotetext{
${ }^{1}$ Department of Stomatology, Huashan Hospital, Fudan University, Shanghai, China; ${ }^{2}$ Institute of Antibiotics, Huashan Hospital, Fudan University, Shanghai, China and ${ }^{3}$ Shanghai Key Laboratory of Stomatology, Department of Prevention, Ninth People's Hospital, Shanghai Jiao Tong University School of Medicine, Shanghai, China

${ }^{4}$ These authors contributed equally to this work.

Correspondence: Professor D Zhu, Institute of Antibiotics, Huashan Hospital, Fudan University, 12M. Wulumuqi Road, Shanghai 200040, China.

E-mail: Zhu_dm@fudan.edu.cn

or Dr L Yu, Department of Stomatology, Huashan Hospital, Fudan University, 12M. Wulumuqi Road, Shanghai 200040, China.

E-mail: wuyu1984@hotmail.com
}

Received 4 June 2012; revised 19 September 2012; accepted 30 September 2012; published online 21 November 2012 
Table1 Susceptibility $(S)$ and resistant $(R)$ rate of 81 anaerobes to six antimicrobial agents $\left(\mu \mathrm{g} \mathrm{ml}^{-1}\right.$ )

\begin{tabular}{|c|c|c|c|c|c|c|c|}
\hline \multirow[b]{2}{*}{ Antimicrobial agents } & \multicolumn{2}{|c|}{ Break point } & \multirow[b]{2}{*}{ MIC ranges } & \multirow[b]{2}{*}{$M I C_{50}$} & \multirow[b]{2}{*}{$M I C_{90}$} & \multirow[b]{2}{*}{$\mathrm{S} \%$} & \multirow[b]{2}{*}{$R \%$} \\
\hline & S & $\mathrm{R}$ & & & & & \\
\hline Cefoxitin & $\leqslant 16$ & $\geqslant 64$ & $0.25-32$ & 4 & 16 & 88.2 & 0 \\
\hline Cefmetazole & $\leqslant 16$ & $\geqslant 64$ & $0.125-64$ & 2 & 8 & 88.2 & 11.8 \\
\hline Imipenem & $\leqslant 4$ & $\geqslant 16$ & $\leqslant 0.06-4$ & 0.5 & 4 & 100 & 0 \\
\hline
\end{tabular}

resistance, such as the involvement of the nim gene, which mediates metronidazole resistance. ${ }^{17-19}$ According to the patterns of susceptibility to six antimicrobial agents of 81 isolates, it seems advisable to periodically monitor anaerobic organisms in China and guide empirical therapy for infections caused by such organisms.

\section{ACKNOWLEDGEMENTS}

This work was supported by Shanghai leading academic discipline project (project number: s30206) and the science and technology commission of Shanghai (08DZ2271100).

1 Meng, H. X. Periodontal abscess. Ann. Periodontol. 4, 79-83 (1999).

2 Cao, C. F., Wu, Y. F. \& Wu, Z. F. Periodontics 146-148 (People‘s Medical Publishing House, Beijing, 2003).

3 Ardila, C. M., Granada, M. I. \& Guzman, I. C. Antibiotic resistance of subgingival species in chronic periodontitis patients. J. Periodont. Res. 45, 557-563 (2010).

4 Singer, E., Calvet, L. \& Mory, F. [Monitoring of antibiotic resistance of gram negative anaerobes]. Méd. Mal. Infect. 38, 256-263 (2008).

5 Behra-Miellet, J. et al. Antibiotic resistance among Gram-negative bacilli: lessons from a French multicentric survey. Anaerobes 9, 105-111 (2003).

6 Hecht, D. W. Prevalence of antibiotic resistance in anaerobic bacteria: worrisome developments. Clin. Infect. Dis. 39, 92-97 (2004).

7 Xu, S. Z., Ma, J. P. \& Ma, L. Y. In vitro antimicrobial susceptibility testing of anaerobic bacteria. J. Clin. Exper. Med. 1, 58-62 (2005).

8 NCCLS. Methods for Antimicrobial Susceptibility Testing of Anaerobic Bacteria. Approved Standard-Fifth Edition 21, M11-A5 (2001).
9 Jaramillo, A. et al. Clinical and microbiological characterization of periodontal abscesses. J. Clin. Periodontol. 32, 1213-1218 (2005).

10 Speer, C., Pelz, K., Hopfenmuller, W. \& Holtgrave, E. A. Investigations on the influencing of the subgingival microflora in chronic periodontitis. J. Orofacial. Orthopedics. 65, 34-47 (2004).

11 Leblbeicioglu, B., Kulekci, G., Ciftci, S., Keskin, F. \& Badur, S. Salivary detection of periodontopathic bacteria and periodontal health status in dental students. Anaerobe 15, 82-86 (2009).

12 Vanwinkelhoff, A. J., Carlee, A. W. \& Degraaff, J. Bacteroides endodontalis and other black-pigmented Bacteroides species in odontogenic abscesses. Infect. Immun. 49, 494-497 (1985)

13 Katsandria, A. et al. In vitro activities of tigecycline against recently isolated Gramnegative anaerobic bacteria in Greece, including metronidazole-resistant strains. Diagn. Microbiol. Infect. Dis. 55, 231-236 (2006).

14 Ulger, N., Celik, C., Cakici, O. \& Soyletir, G. Antimicrobial susceptibilities of Bacteroides fragilis and Bacteroides thetaiotaomicron strains isolated from clinical specimens and human intestinal microbiota. Anaerobe 10, 255-259 (2004).

15 Aldridge, K. E. et al. Multicenter survey of the changing in vitro antimicrobia susceptibilities of clinical isolates of Bacteroides fragilis group, Prevotella, Fusobacterium, Porphyromonas, and Peptostreptococcus species. Antimicrob. Agents Chemother. 45, 1238-1243 (2001).

16 Maestre, J. R. et al. Odontogenic bacteria in periodontal disease and resistance patterns to common antibiotics used as treatment and prophylaxis in odontology in Spain. Rev. Esp. Quimioter. 20, 61-67 (2007).

17 Alauzet, C. et al. Metronidazole resistance in Prevotella spp. and description of a new nim gene in Prevotella baroniae. Antimicrob. Agents Chemother. 54, 60-64 (2010).

18 Carlier, J. P., Sellier, N., Ranger, M. N. \& Reysset, G. Metabolism of a 5-nitroimidazole in susceptible and resistant isogenic strains of Bacteroides fragilis. Antimicro. Agents Chemo. 41, 14951499 (1997).

19 Gal, M. \& Brazier, J. S. Metronidazole resistance in Bacteroides spp. carrying nim genes and the selection of slow-growing metronidazole-resistant mutants. J. Antimicrob. Chemother. 54, 109-116 (2004). 